\title{
THE CENTRAL REGION OF NGC 1365. SEST AND VLA OBSERVATIONS OF CO AND THE RADIO CONTINUUM
}

\author{
AA. SANDQVIST, S. JÖRSÄTER and P. O. LINDBLAD \\ Stockholm Observatory, S-139 36 Saltsjöbaden, Sweden
}

\begin{abstract}
The barred spiral Seyfert galaxy NGC 1365 has been observed in the radio continuum at wavelengths of 2,6 and $20 \mathrm{~cm}$, using scaled arrays of the VLA, and complete maps have been made in the $J=1-0$ and $J=2-1$ CO emission lines using the SEST. MEM maps of the 6 and $20 \mathrm{~cm}$ emission, as well as a spectral index map, have been produced with a resolution of $2^{\prime \prime} .3 \times 0$ " .9, and the $2-\mathrm{cm}$ map has a resolution of $0^{\prime \prime} .25 \times 0^{\prime \prime} .10$. The dominant continuum features are a number of unresolved sources with relatively flat non-thermal spectral indices $(-0.3$ to -0.5$)$, immersed in an incomplete circumnuclear ring, which is superimposed upon a background that extends into the bar along the prominent dust lanes. The ring has angular dimensions of $8^{\prime \prime} \times 20^{\prime \prime}$, which corresponds to a linear dimension of the order of $1 \mathrm{kpc}$. There is clear evidence of a jet, about 5" long, originating at the position of the Seyfert nucleus and extending in a southeastern direction, closely along the minor axis of the galaxy. The jet has a steep non-thermal spectral index $(-1.0)$ and is aligned along the axis of a conical shell of [OIII] emission. The $\mathrm{CO}$ molecular gas peaks at the nucleus and is strongly concentrated to the nucleus and bar regions with a certain enhancement along the bar. The total molecular hydrogen gas mass in the observed region is $2 \times 10^{10} M_{\odot}$, with $6 \times 10^{9} M_{\odot}$ lying within $2.2 \mathrm{kpc}$ of the nucleus. A full presentation of the results will be published in Astronomy and Astrophysics in 1994.
\end{abstract}
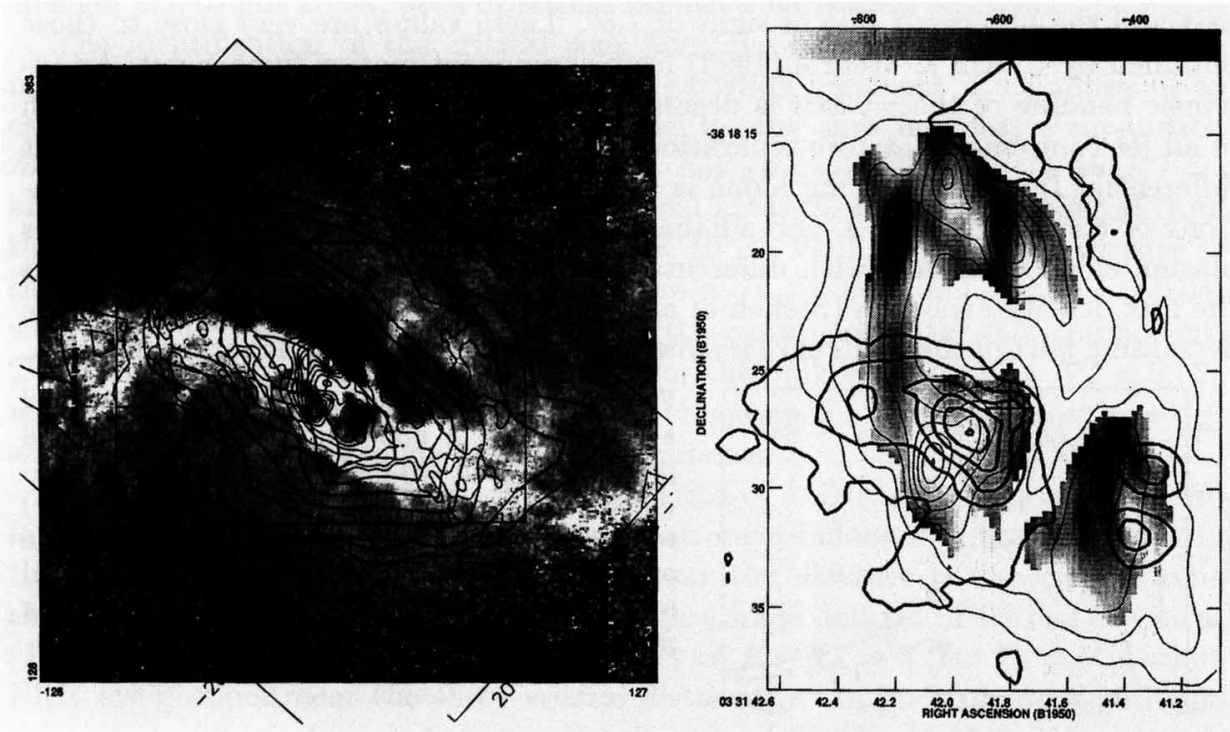

Fig. 1. NGC 1365. Bar Region (left): $J=2-1 \mathrm{CO} T_{\mathrm{A}}^{*}$-thick lines; $20 \mathrm{~cm}$ continuum-thin lines; $B$ - Gunn $z$ colour index-grays. Central Region (right): [OIII-thick lines; $20 \mathrm{~cm}$ continuum-thin lines; spectral index-grays; nucleus-cross.

T. J.-L. Courvoisier and A. Blecha: Multi-Wavelength Continuum Emission of AGN, 431.

(C) 1994 IAU. Printed in the Netherlands. 